\title{
A LYAPUNOV TYPE INEQUALITY FOR INDEFINITE WEIGHTS AND EIGENVALUE HOMOGENIZATION
}

\author{
JULIÁN FERNÁNDEZ BONDER, JUAN P. PINASCO, ARIEL M. SALORT
}

\begin{abstract}
In this paper we prove a Lyapunov type inequality for quasilinear problems with indefinite weights. We show that the first eigenvalue is bounded below in terms of the integral of the weight, instead of the integral of its positive part. We apply this inequality to some eigenvalue homogenization problems with indefinite weights.
\end{abstract}

\section{INTRODUCTION}

In this paper we will consider the following quasilinear eigenvalue problem,

$$
\left\{\begin{array}{l}
-\left(a(x)\left|u^{\prime}\right|^{p-2} u^{\prime}\right)^{\prime}=\lambda \rho(x)|u|^{p-2} u \quad x \in(0, L) \\
u(0)=u(L)=0,
\end{array}\right.
$$

where $1<p<\infty, \lambda$ is a real parameter, the weight $\rho \in L^{1}(0, L)$ is allowed to change signs, and the coefficient $a(x)$ belongs to the Muckenhoupt class $\mathcal{A}_{p}$, see Section $\S 2$ for definitions.

Quasilinear eigenvalue problems with indefinite weighs were studied first by Zeidler in [25], with $a \equiv 1$, and the extension to general coefficients $a \in \mathcal{A}_{p}$ follows by using the techniques in [17] and [22]. There are several results concerning the existence and asymptotic behavior of two sequences of eigenvalues $\left\{\lambda_{k}^{+}\right\}_{k \geq 1},\left\{\lambda_{k}^{-}\right\}_{k \geq 1}$, going to $\pm \infty$, in both the linear and nonlinear case, in one and several dimensions, see for instance $[1,5,7,10,14,21]$.

For one dimensional problems, the eigenvalues are simple, in the sense that $\lambda_{k}^{ \pm}$has a unique (up to a multiplicative constant) associated eigenfunction $u_{k}^{ \pm}$with exactly $k+1$ zeros in $[0, L]$. When $a \equiv 1$, the asymptotic behavior of the eigenvalues is given by

$$
\lambda_{k}^{ \pm} \sim\left(\frac{\pi_{p}}{\int_{0}^{L}\left(\rho^{ \pm}(x)\right)^{1 / p} d x}\right)^{p},
$$

where $\rho^{+}(x)=\max \{\rho(x), 0\}$, and $\rho^{-}=(-\rho)^{+}$, and

$$
\pi_{p}=2(p-1)^{1 / p} \int_{0}^{1} \frac{d s}{\left(1-s^{p}\right)^{1 / p}} .
$$

The case of nonconstant principal coefficient can be analyzed easily in exactly the same way by using a change of variable (see Section $\S 2$ ). 
However, the asymptotic formula (1.2) cannot be used to derive precise bounds for $\lambda_{k}^{ \pm}$. A useful tool in order to obtain lower bounds for eigenvalues in one dimensional problems is Lyapunov's inequality: if there exists a nontrivial solution $u$ of

$$
\left\{\begin{array}{l}
-u^{\prime \prime}=\rho(x) u \quad x \in(0, L) \\
u(0)=u(L)=0,
\end{array}\right.
$$

then we must have

$$
\frac{4}{L} \leq \int_{0}^{L}|\rho(t)| d t
$$

an inequality proved by Borg [2], and improved later by Wintner [24] who used $\rho^{+}(t)$ instead of $|\rho(t)|$. A similar inequality holds for $p$-Laplacian operators, and applying it in each nodal domain, we can bound the $k$-th eigenvalue as follows,

$$
\frac{2^{p} k^{p}}{L^{p-1}} \leq \lambda_{k} \int_{0}^{L} \rho^{+}(t) d t
$$

see [19], and [20] for a recent survey.

However, it is possible to obtain better bounds of the eigenvalues, and we will prove the following Lyapunov type inequality which takes care of the negative part of the weight as well:

Theorem 1.1. Let $u$ be a solution of

$$
-\left(a(x)\left|u^{\prime}\right|^{p-2} u^{\prime}\right)^{\prime}=\rho(x)|u|^{p-2} u \quad x \in(0, L)
$$

satisfying the boundary condition $u(0)=u(L)=0$. Then,

$$
\frac{1}{p}\left(\int_{0}^{L} a^{-\frac{1}{p-1}}(t) d t\right)^{1-p} \leq \sup _{0 \leq x \leq L}\left|\int_{0}^{x} \rho(t) d t\right|
$$

As a consequence, we have the following bound for $\lambda_{k}^{+}$:

Theorem 1.2. Let $\lambda_{k}^{+}$be the $k$-th eigenvalue of problem (1.1) with $\rho \in L^{1}[0, L]$. Then,

$$
\frac{k^{p-1}}{p}\left(\int_{0}^{L} a^{-\frac{1}{p-1}}(t) d t\right)^{1-p} \leq \lambda_{k} \cdot \sup _{(a, b) \subset[0, L]}\left|\int_{a}^{b} \rho(t) d t\right|
$$

Let us mention that inequalities (1.4) and (1.5) are closely related to the ones introduced by Harris and Kong in [12] and generalized in [13] for quasilinear problems like (1.1) with $a \equiv 1$. However, a mixed boundary condition was involved in these inequalities: suppose that $u^{\prime}(0)=u(L)=0$, then

$$
1 \leq L^{p-1} \sup _{0 \leq x \leq L} \int_{0}^{x} \rho(t) d t
$$

and, for $u(0)=u^{\prime}(L)=0$,

$$
1 \leq L^{p-1} \sup _{0 \leq x \leq L} \int_{x}^{L} \rho(t) d t
$$

These inequalities were proved by using Ricatti equation techniques, that is, by introducing the function $v=u^{\prime} / u$, and studying a first order nonlinear differential 
equation for $v$, with the initial condition $v(0)=0$ and using the fact that $v$ blows up when $x \rightarrow L$.

The main drawback of this approach is that it cannot be used for higher order equations, so our proofs of Theorems 1.1, 1.2 and 1.3 are based on variational arguments, and we give the following extension to higher order differential equations:

Theorem 1.3. Let $u$ be a solution of

$$
\begin{cases}(-1)^{m} u^{(2 m)}=\rho(x) u & x \in(0, L) \\ u^{(j)}(0)=u^{(j)}(L)=0 & 0 \leq j \leq m\end{cases}
$$

where $m \geq 1$ and $\rho \in L^{1}[0, L]$. Then,

$$
\frac{(m-1)[(m-2) !]^{2}}{2 L^{2 m-1}} \leq \sup _{0 \leq x \leq L} \int_{0}^{x} \rho(t) d t
$$

With the same ideas we will prove a version of Theorem 1.3 including higher order quasilinear equations.

Remark 1.4. When $p=2, a \equiv 1$, and $\rho \geq 0$, the constant obtained by Das and Vatsala in [6] for the classical Lyapunov's inequality is given by

$$
\frac{24^{2 m-1}(2 m-1)[(m-2) !]^{2}}{2 L^{2 m-1}} .
$$

For $p \neq 2$ and $m>1$ the determination of the best constant remains open even for the classical Lyapunov's inequality with positive weights, see [23].

Although the constant in Theorem 1.3 is worse than the one in the classical inequality, we will see in the applications that the cancelation of the positive and negative parts of the weight $\rho$ in the integral gives much better bounds in several cases. Hence, the true contribution of this kind of inequalities cames from the right-hand side.

Let us focus now in applications of Theorem 1.2. Let us consider first the following periodic homogenization problem

$$
\left\{\begin{array}{l}
-\left(a\left(\frac{x}{\varepsilon}\right)\left|u_{\varepsilon}^{\prime}\right|^{p-2} u_{\varepsilon}^{\prime}\right)^{\prime}=\lambda_{\varepsilon} \rho\left(\frac{x}{\varepsilon}\right)\left|u_{\varepsilon}\right|^{p-2} u_{\varepsilon} \quad x \in(0, L) \\
u_{\varepsilon}(0)=u_{\varepsilon}(L)=0
\end{array}\right.
$$

where $\varepsilon$ is a positive small parameter, and we assume that $a, \rho$ are $L$-periodic functions. For each fixed $\varepsilon$, the results for problem (1.1) hold: we have two sequences of simple eigenvalues $\left\{\lambda_{\varepsilon, k}^{ \pm}\right\}_{k \geq 1}$, and their corresponding eigenfunctions satisfy the same nodal properties as before.

We are interested in the behavior of $\left\{\lambda_{\varepsilon, k}^{ \pm}\right\}_{\varepsilon}$ when $\varepsilon \rightarrow 0^{+}$for each $k$ fixed. For nonnegative weights $\rho$, it is known (see $[3,8,9]$ ) that $\lambda_{\varepsilon, k} \rightarrow \lambda_{0, k}$ when $\varepsilon \rightarrow 0^{+}$, where $\lambda_{0, k}$ is the $k$-th eigenvalue of the limit problem

$$
\left\{\begin{array}{l}
-\left(a^{*}\left|u^{\prime}\right|^{p-2} u^{\prime}\right)^{\prime}=\lambda_{0} \bar{\rho}|u|^{p-2} u \quad x \in(0, L) \\
u(0)=u(L)=0,
\end{array}\right.
$$


where $\bar{\rho}$ is the average of $\rho$ in the interval $(0, L)$ and $a^{*}$ is given by

$$
a^{*}:=\left(\int_{0}^{L} a(t)^{-\frac{1}{p-1}} d t\right)^{1-p}
$$

see [9] for a proof. Remarkably, observe that $a^{*}$ is the constant appearing in Lyapunov's inequality (1.4).

We may ask what happens when $\rho$ is an indefinite weight, and our next Theorem generalizes to the one dimensional quasilinear setting the answer for second order linear problems (in arbitrary spatial dimension) obtained recently by Nazarov, Pankratova and Piatnitski in [16, 18]:

Theorem 1.5. Let $\left\{\lambda_{\varepsilon, k}^{ \pm}\right\}_{k \geq 1}$ be the eigenvalues of problem (1.8), and $\left\{\lambda_{0, k}\right\}_{k \geq 1}$ be the eigenvalues of problem (1.9). Then,

(1) If $\bar{\rho}=0$ then $\lambda_{\varepsilon, k}^{ \pm} \rightarrow \pm \infty$ as $\varepsilon \rightarrow 0^{+}$.

(2) If $\bar{\rho}<0$ then $\lambda_{\varepsilon, k}^{-} \rightarrow \lambda_{0, k}$ and $\lambda_{\varepsilon, k}^{+} \rightarrow+\infty$ as $\varepsilon \rightarrow 0^{+}$.

(3) If $\bar{\rho}>0$ then $\lambda_{\varepsilon, k}^{+} \rightarrow \lambda_{0, k}$ and $\lambda_{\varepsilon, k}^{-} \rightarrow-\infty$ as $\varepsilon \rightarrow 0^{+}$.

Let us remark that in the first case, after a suitable renormalization $\mu_{\varepsilon, k}^{ \pm}=$ $\varepsilon^{\alpha} \lambda_{\varepsilon, k}^{ \pm}$, the convergence to the eigenvalues of a different limit problem was obtained in $[16,18]$. Their proofs were based on linear tools such as orthogonality of eigenfunctions or asymptotic expansions in powers of $\varepsilon$ which are not available here.

The paper is organized as follows: In Section $\S 2$ we introduce some necessary facts about the eigenvalue problem for the $p$-Laplace operator. In Section $\S 3$ we prove Theorems 1.1, 1.2 and 1.3. Section $\S 4$ is devoted to the proof of Theorem 1.5 .

\section{Preliminary Results}

2.1. Eigenvalues of $p$-Laplacian operators. The eigenvalue problem for the $p$ Laplacian operator started with the pioneering results of Browder in the $1960 \mathrm{~s}$. A recent survey including more general quasilinear homogeneous operators in $\Omega \subset R^{N}$ can be found in [9]. We will need here the following variational characterization of eigenvalues:

Theorem 2.1. There exists a double sequence of variational eigenvalues $\left\{\lambda_{k}^{ \pm}\right\}_{k \geq 1}$ of problem (1.1), given by

$$
\lambda_{k}^{ \pm}=\inf _{C \in \mathcal{C}_{k}} \sup _{u \in C} \int_{0}^{L} a(x)\left|u^{\prime}\right|^{p} d x
$$

where

$$
\begin{aligned}
\mathcal{C}_{k} & =\left\{C \subset M^{ \pm}: C \text { is compact, } C=-C, \gamma(C) \geq k\right\}, \\
M^{ \pm} & =\left\{u \in W_{0}^{1, p}(0, L): \int_{0}^{L} \rho(x)|u|^{p} d x= \pm 1\right\} .
\end{aligned}
$$

and $\gamma$ is the Krasnoselskii genus. 
The proof is identical to the one in [11] for positive weights.

Remark 2.2. Sometimes we will work with an equivalent definition (due to the homogeneity of the operator),

$$
\lambda_{k}^{ \pm}=\inf _{C \in \mathcal{C}_{k}} \sup _{u \in C} \frac{\int_{0}^{L} a(x)\left|u^{\prime}\right|^{p} d x}{\int_{0}^{L} \rho(x)|u|^{p}},
$$

where now

$$
\begin{aligned}
& M^{+}=\left\{u \in W_{0}^{1, p}(0, L): \int_{0}^{L} \rho(x)|u|^{p} d x>0\right\}, \\
& M^{-}=\left\{u \in W_{0}^{1, p}(0, L): \int_{0}^{L} \rho(x)|u|^{p} d x<0\right\} .
\end{aligned}
$$

We need also the following results from the Sturm-Liouville theory for the p-Laplacian:

Theorem 2.3. Let $\left\{u_{k}^{ \pm}\right\}_{k \geq 1}$ be a sequence of normalized eigenfunctions corresponding to $\left\{\lambda_{k}^{ \pm}\right\}_{k \geq 1}$. Then, $u_{k}^{ \pm}$has exactly $k+1$ zeros in $[0, L]$ which determine $k$ nodal domains.

Theorem 2.4. Let $\rho_{1}, \rho_{2} \in L^{1}(0, L)$, with $\rho_{1}(x) \leq \rho_{2}(x)$. Then,

$$
\begin{aligned}
& \lambda_{k}^{+}\left(\rho_{1}\right) \geq \lambda_{k}^{+}\left(\rho_{2}\right), \\
& \lambda_{k}^{-}\left(\rho_{1}\right) \leq \lambda_{k}^{-}\left(\rho_{2}\right),
\end{aligned}
$$

whenever $\lambda_{k}^{ \pm}\left(\rho_{1}\right)$ and $\lambda_{k}^{ \pm}\left(\rho_{2}\right)$ exists.

The proof is a consequence of the alternative variational characterization (2.2)), by observing that $M^{+}\left(\rho_{2}\right) \subset M^{+}\left(\rho_{1}\right)$, and after changing $\rho \rightarrow-\rho$ the other follows as well. Clearly, if one weight is negative, and the other one is positive, there are no eigenvalues to compare.

2.2. The Muckenhoupt class of $A_{p}$ weights. We say that $a$ belongs to the Muckenhoupt $\mathcal{A}_{p}$-class if $a$ is a nonnegative function in $L_{l o c}^{1}\left(\mathbb{R}^{N}\right)$, such that

$$
\left(\int_{B} a(x) d x\right)\left(\int_{B} a(x)^{-\frac{1}{p-1}} d x\right)^{p-1} \leq c_{p, a}|B|^{p}
$$

for any ball $B \in \mathbb{R}^{N}$, and a fixed positive constant $c_{p, a}$.

Remark 2.5. Beside the fact that the bounds we will study below depend strongly on the existence of the second integral in equation (2.3), the most important fact is that $\mathcal{A}_{p}$ weights guarantee the density of smooth functions in $W^{1, p}(\Omega)$, see $[15,22]$, and is the right class of coefficients involved in different quasilinear problems. The $\mathcal{A}_{p}$ class contains several interesting weights, like powers of the distance to a point or the distance to the boundary, allowing both singular and degenerate coefficients.

We can avoid the coefficient $a$ in problem (1.1) by performing a change of variables. If we define

$$
P(x)=\int_{0}^{x} \frac{1}{a(s)^{1 /(p-1)}} d s,
$$


and perform the change of variables

$$
(x, u) \rightarrow(y, v)
$$

where

$$
y=P(x), \quad v(y)=u(x),
$$

a simple computation gives

$$
\left\{\begin{array}{l}
-\left(|\dot{v}|^{p-2} \dot{v}\right)=\lambda Q(y)|v|^{p-2} v, \quad y \in[0, \ell] \\
v(0)=v(\ell)=0
\end{array}\right.
$$

where

$$
\cdot=d / d y
$$

with

$$
\ell=\int_{0}^{1} \frac{1}{a(s)^{1 /(p-1)}} d s \rightarrow \ell=\overline{a^{\frac{-1}{p-1}}}
$$

and

$$
\begin{aligned}
Q(y) & =a(x)^{1 /(p-1)} \rho(x) \\
& =a\left(P^{-1}(y)\right)^{1 /(p-1)} \rho\left(P^{-1}(y)\right) .
\end{aligned}
$$

Both $P$ and $\ell$ are well defined whenever $a \in \mathcal{A}_{p}$.

\section{A Lyapunov type ineQuALity}

We prove in this section our main results. Before the proofs, let us state and prove the following useful lemma:

Lemma 3.1. Let $1<p<\infty$, and let $u \in C_{0}^{(m)}(0, L)$, with

$$
u^{j}(a)=0, \quad 0 \leq j \leq m-1,
$$

and $a(x) \in A_{p}$. Then,

$$
\|u\|_{L^{\infty}(0, L)} \leq \frac{L^{m-1}}{(m-1) !}\left(\int_{0}^{L} a^{-\frac{1}{p-1}}(t) d t\right)^{\frac{p-1}{p}}\left(\int_{0}^{L} a(t)\left|u^{(m)}(t)\right|^{p} d t\right)^{\frac{1}{p}} .
$$

Proof. We apply Taylor's expansion and the Cauchy expression for the remainder, obtaining

$$
u(x)=\sum_{j=0}^{m-1} \frac{u^{j}(0)}{j !} x^{j}+\int_{0}^{x} \frac{(x-t)^{m-1}}{(m-1) !} u^{(m)}(t) d t .
$$

However, since the derivatives of $u$ at 0 are zero, we have

$$
u(x)=\int_{0}^{x} \frac{(x-t)^{m-1}}{(m-1) !} u^{m}(t) d t .
$$


Thus, bounding $x-t<L$, and by using Holder's inequality,

$$
\begin{aligned}
|u(x)| & \leq L^{m-1} \int_{0}^{x} a^{\frac{1}{p}}(x) a^{-\frac{1}{p}}(x) \frac{u^{(m)}(t)}{(m-1) !} d t \\
& \leq L^{m-1}\left(\int_{0}^{x} a^{-\frac{1}{p-1}}(x) d t\right)^{\frac{p-1}{p}}\left(\int_{0}^{x} a(x)\left|\frac{u^{(m)}(t)}{(m-1) !}\right|^{p} d t\right)^{\frac{1}{p}},
\end{aligned}
$$

and the inequality is proved.

Proof of Theorem 1.1. By multiplying equation (1.3) by $u$, and integrating by parts we get

$$
\int_{0}^{L} a(x)\left|u^{\prime}\right|^{p} d x=\int_{0}^{L} \rho(x)|u|^{p} d x
$$

Let us introduce now the function $Q(x)=\int_{0}^{x} \rho(t) d t$. Hence,

$$
\int_{0}^{L} \rho(x)|u|^{p} d x=\int_{0}^{L} Q^{\prime}(x)|u|^{p} d x=-\int_{0}^{L} Q(x) p|u|^{p-2} u u^{\prime} d x .
$$

By combining these with Lemma 3.1, for $m=1$, we obtain

$$
\begin{aligned}
\int_{0}^{L} a(x)\left|u^{\prime}\right|^{p} d x & =\int_{0}^{L} \rho(x)|u|^{p} d x \\
& \leq p \sup _{0 \leq x \leq L}|Q|\|u\|_{L^{\infty}(0, L)}^{p-1} \int_{0}^{L}\left|u^{\prime}\right| d x \\
& \leq p \sup _{0 \leq x \leq L}|Q|\left(\int_{0}^{L} a^{-\frac{1}{p-1}}(t) d t\right)^{\frac{(p-1)^{2}}{p}} \\
& \times\left(\int_{0}^{L} a(t)\left|u^{\prime}(t)\right|^{p} d t\right)^{\frac{p-1}{p}} \int_{0}^{L}\left|u^{\prime}\right| d x .
\end{aligned}
$$

Now, by inserting $a^{\frac{1}{p}}(x) a^{-\frac{1}{p}}(x)$ in the last integral, and using again Holder's inequality, we get after rearranging,

$$
\int_{0}^{L} a(x)\left|u^{\prime}\right|^{p} d x \leq p \sup _{0 \leq x \leq L}|Q|\left(\int_{0}^{L} a^{-\frac{1}{p-1}}(t) d t\right)^{p-1} \int_{0}^{L} a(t)\left|u^{\prime}(t)\right|^{p} d t
$$

and, after canceling the integral in both sides, the theorem is proved.

In the next proof we use that the eigenfunction $u_{k}$ associated to $\lambda_{k}$ has exactly $k$ nodal domains.

Proof of Theorem 1.2. Let us fix $k \geq 1$, and let $0=x_{0}<x_{1}<\cdots<x_{n}=L$ be the $k+1$ zeros of the associated eigenfunction $u_{k}$. We can apply Lemma 1.3 in each 
nodal domain $\left(x_{i-1}, x_{i}\right)$, obtaining

$$
\frac{1}{p^{\frac{1}{p-1}}} \leq \lambda_{k}^{\frac{1}{p-1}}\left(\sup _{x_{i-1} \leq x \leq x_{i}}\left|\int_{x_{i-1}}^{x} \rho(t) d t\right|\right)^{\frac{1}{p-1}} \cdot \int_{x_{i-1}}^{x_{i}} a^{-\frac{1}{p-1}}(t) d t,
$$

and therefore,

$$
\begin{aligned}
\frac{k}{p^{\frac{1}{p-1}}} & \leq \lambda_{k}^{\frac{1}{p-1}} \sum_{i=1}^{k}\left(\sup _{x_{i-1} \leq x \leq x_{i}}\left|\int_{x_{i-1}}^{x} \rho(t) d t\right|\right)^{\frac{1}{p-1}} \cdot \int_{x_{i-1}}^{x_{i}} a^{-\frac{1}{p-1}}(t) d t \\
& \leq \lambda_{k}^{\frac{1}{p-1}}\left(\sup _{(a, b) \subset[0, L]}\left|\int_{a}^{b} \rho(t) d t\right|\right)^{\frac{1}{p-1}} \cdot \sum_{i=1}^{k} \int_{x_{i-1}}^{x_{i}} a^{-\frac{1}{p-1}}(t) d t .
\end{aligned}
$$

Hence,

$$
\frac{1}{p}\left(\frac{k}{\int_{0}^{L} a^{-\frac{1}{p-1}}(x) d t}\right)^{p-1} \leq \lambda_{k} \sup _{(a, b) \subset[0, L]}\left|\int_{a}^{b} \rho(t) d t\right|
$$

and the proof is finished

The proof of Theorem 1.3 is similar. Let us prove the following result which is more general:

Theorem 3.2. Let $u \in W_{0}^{m, p}(0, L)$ be a nontrivial solution of

$$
\left\{\begin{array}{l}
(-1)^{m}\left(a(x)\left|u^{(m)}\right|^{p-2} u^{(m)}\right)^{(m)}=\rho(x) u \\
u^{(j)}(0)=u^{(j)}(L)=0 \quad 0 \leq j \leq m,
\end{array}\right.
$$

where $m \geq 1, a \in \mathcal{A}_{p}$, and $\rho \in L^{1}$. Then

$$
\frac{(m-1)^{p-1}[(m-2) !]^{p}}{p L^{m p-p}}\left(\int_{0}^{L} a^{-\frac{1}{p-1}}(x) d t\right)^{1-p} \leq \sup _{0 \leq x \leq L} \int_{0}^{x} \rho(t) d t .
$$

Proof. We start from

$$
\begin{aligned}
\int_{0}^{L} a(x)\left|u^{(m)}\right|^{p} d x & =\int_{0}^{L} Q(x)^{\prime}|u|^{p} d x \\
& =-\int_{0}^{L} Q(x) p|u|^{p-2} u u^{\prime} d x \\
& \leq p \sup _{0 \leq x \leq L}|Q| \int_{0}^{L}|u|^{p-1}\left|u^{\prime}\right| d x
\end{aligned}
$$

with $Q=\int_{0}^{x} \rho(t) d t$ as before. 
Let us call $A=\|u\|_{L^{\infty}}^{p-1}$ and $B=\left\|u^{\prime}\right\|_{L^{\infty}}$. Since $u \in W_{0}^{m, p}$ implies that $u^{\prime} \in$ $W_{0}^{m-1, p}$, using Lemma 3.1, we have

$$
\begin{aligned}
& A \leq\left[\frac{L^{m-1}}{(m-1) !}\left(\int_{0}^{L} a^{-\frac{1}{p-1}}(x) d t\right)^{\frac{p-1}{p}}\left(\int_{0}^{L} a(x)\left|u^{(m)}(t)\right|^{p} d t\right)^{\frac{1}{p}}\right]^{p-1} . \\
& B \leq \frac{L^{m-2}}{(m-2) !}\left(\int_{0}^{L} a^{-\frac{1}{p-1}}(x) d t\right)^{\frac{p-1}{p}}\left(\int_{0}^{L} a(x)\left|u^{(m)}(t)\right|^{p} d t\right)^{\frac{1}{p}} .
\end{aligned}
$$

Now, we can bound

$$
\begin{aligned}
\int_{0}^{L}|u|^{p-1}\left|u^{\prime}\right| d x & \leq A \cdot B \cdot L \\
& =C\left(\int_{0}^{L} a^{-\frac{1}{p-1}}(x) d t\right)^{p-1} \int_{0}^{L} a(x)\left|u^{(m)}(t)\right|^{p} d t
\end{aligned}
$$

where

and the result follows.

$$
C=\frac{L^{m p-p}}{(m-1)^{p-1}[(m-2) !]^{p}},
$$

Remark 3.3. When $p=2$, and $a \equiv 1$, we get

$$
\frac{(m-1)[(m-2) !]^{2}}{2 L^{2 m-1}} \leq \sup _{0 \leq x \leq L} \int_{0}^{x} \rho(t) d t
$$

as in Theorem 1.3.

\section{The homogenization PRoblem}

Proof of Theorem 1.5. We prove only the assertions for the positive eigenvalues $\left\{\lambda_{\varepsilon, k}^{+}\right\}$, for the negative ones the result follows by considering the weight $-\rho$.

(1) Let $\rho \in L^{1}$ be an $L$-periodic function with zero mean. Then, a simple computation gives.

$$
\sup _{(a, b) \subset[0, L]}\left|\int_{a}^{b} \rho\left(\frac{x}{\varepsilon}\right) d t\right| \leq \sup _{(a, b) \subset[0, \varepsilon]} \int_{a}^{b}\left|\rho\left(\frac{x}{\varepsilon}\right)\right| d t \leq \varepsilon\|\rho\|_{L^{1}(0, L)} .
$$

Now, Lyapunov's inequality (1.5) gives

$$
\frac{k^{p-1}}{\varepsilon p\|\rho\|_{L^{1}(0, L)}}\left(\int_{0}^{L} a^{-\frac{1}{p-1}}(t) d t\right)^{1-p} \leq \lambda_{\varepsilon, k}^{+}
$$

which proves that the eigenvalues diverge as $\varepsilon \rightarrow 0^{+}$.

(2) Let us assume that $\bar{\rho}<0$, and let us introduce the weight

$$
\sigma=\rho-\bar{\rho} .
$$


Now, $\sigma>\rho$, and $\bar{\sigma}=0$. So, from from the Sturmian comparison theorem 2.4, and part (1) we get

$$
\lambda_{\varepsilon, k}^{+}(\rho) \geq \lambda_{\varepsilon, k}^{+}(\sigma) \geq \frac{k^{p-1}}{\varepsilon p\|\sigma\|_{L^{1}(0, L)}}\left(\int_{0}^{L} a^{-\frac{1}{p-1}}(t) d t\right)^{1-p},
$$

and the result follows.

(3) Let us assume now that $\bar{\rho}>0$, and let us show that the eigenvalues $\lambda_{\varepsilon, k}^{+}$ convege to $\lambda_{0, k}^{+}$. To this end, we need to show first that $\left\{\lambda_{\varepsilon, k}^{+}\right\}_{\varepsilon}$ is uniformly bounded away from zero and infinity. Then, the remaining steps of the proof are the same as in the case of positive weights, and can be found in $[3,9]$ : we extract a convergent sequence of eigenvalues, we choose a sequence of normalized eigenfunctions corresponding to these eigenfunctions, and Rellich-Kondrachov enable us to extract a convergent subsequence. The convergence of the full family follows from the simplicity of the eigenfuctions of problem (1.9), see [9] for details.

So, we only need to show that $c<\lambda_{\varepsilon, k}^{+}<C$ for some positive constants $c, C$.

The lower bound follows from Lyapunov's inequality (1.5),

$$
\frac{k^{p-1}}{p}\left(\int_{0}^{L} a^{-\frac{1}{p-1}}\left(\frac{t}{\varepsilon}\right) d t\right)^{1-p} \leq \lambda_{\varepsilon, k}^{+} \cdot \sup _{(a, b) \subset[0, L]}\left|\int_{a}^{b} \rho\left(\frac{t}{\varepsilon}\right) d t\right| \leq \lambda_{\varepsilon, k}^{+} \int_{0}^{L}\left|\rho\left(\frac{t}{\varepsilon}\right)\right| d t,
$$

and using that

$$
\int_{0}^{L} f\left(\frac{t}{\varepsilon}\right) d t=\int_{0}^{L} f(t) d t+O(\varepsilon)
$$

for any $L$-periodic function $f \in L^{1}$, we get a lower bound for $\lambda_{\varepsilon, k}^{+}$independently of $\varepsilon$.

Let us find an upper bound of the first eigenvalue. To this end, we construct a test function and compute the Rayleigh quotient (2.2). We choose $h$ small, and we define a continuous function $w$ which grows linearly from zero to 1 in $[0, h]$, remains constant in $[h, L-h]$, and decreases linearly from 1 to zero in $[L-h, L]$. Now, we have

$$
\begin{aligned}
\int_{0}^{L} a\left(\frac{t}{\varepsilon}\right)\left|w^{\prime}\right|^{p} & \leq 2 h^{-p} \int_{0}^{L} a\left(\frac{t}{\varepsilon}\right) d t \\
& \leq 4 L h^{-p}\|a\|_{L^{1}([0, L])} \\
\int_{0}^{L} \rho\left(\frac{x}{\varepsilon}\right)|w|^{p} d x & \geq\left(\left\lfloor\frac{L-2 h}{\varepsilon}\right\rfloor-2\right) \varepsilon L \int_{0}^{L} \rho(t) d t-2\left(\left\lfloor\frac{h}{\varepsilon}\right\rfloor+2\right) \varepsilon L \int_{0}^{L}|\rho(t)| d t \\
& \geq \frac{L^{3}}{2} \bar{\rho},
\end{aligned}
$$

for $h$ fixed sufficiently small and any $\varepsilon<\varepsilon_{0}(h)$.

Therefore,

$$
\lambda_{\varepsilon, 1}^{+} \leq \frac{8\|a\|_{L^{1}([0, L])}}{h^{p} L^{2} \bar{\rho}},
$$

and the first eigenvalue is bounded above. 
The same argument can be applied for higher eigenvalues. In fact let $w$ be the previously defined function and introduce $k$ functions $\left\{w_{i}\right\}_{1 \leq i \leq k}$ defined on $[0, L]$, where

$$
w_{i}(x)= \begin{cases}w(k x-(i-1) L) & x \in[(i-1) L / k, i L / k] \\ 0 & x \in[0, L] \backslash[(i-1) L / k, i L / k]\end{cases}
$$

We consider the set $C=\operatorname{span}\left\{w_{1}, \ldots, w_{k}\right\} \cap S$, where $S$ is the unit ball in $W_{0}^{1, p}([0, L])$. Since $\gamma(C)=k$, and $C \subset M^{+}(\rho)$ for $\varepsilon<\varepsilon_{0}(k)$, it is an admissible set in the characterization of $\lambda_{\varepsilon, k}^{+}$. Thus, if $v=\sum c_{i} w_{i} \in C$, we have $\sum_{i=1}^{k}\left|c_{i}\right|^{p}=1$ and

$$
\begin{aligned}
\int_{0}^{L} a\left(\frac{x}{\varepsilon}\right)\left|v^{\prime}\right|^{p} d x & \leq 2 h^{-p} k^{p} \int_{0}^{L} a\left(\frac{x}{\varepsilon}\right) d x \sum_{i=1}^{k}\left|c_{i}\right|^{p} \\
& \leq 4 L h^{-p} k^{p}\|a\|_{L^{1}([0, L])} \\
\int_{0}^{L} \rho\left(\frac{x}{\varepsilon}\right)|v|^{p} d x & =\sum_{i=1}^{k}\left|c_{i}\right|^{p} \int_{0}^{L} \rho\left(\frac{x}{\varepsilon}\right)\left|w_{i}\right|^{p} d x \\
& \geq \sum_{i=1}^{k}\left|c_{i}\right|^{p} \frac{L^{3}}{k^{3} 2} \bar{\rho} \\
& =\frac{L^{3}}{2 k^{3}} \bar{\rho} .
\end{aligned}
$$

and we get

$$
\lambda_{\varepsilon, k}^{+}=\inf _{C \in \mathcal{C}_{k}} \sup _{v \in C} \frac{\int_{0}^{L} a\left(\frac{x}{\varepsilon}\right)\left|v^{\prime}\right|^{p} d x}{\int_{0}^{L} \rho\left(\frac{x}{\varepsilon}\right)|v|^{p} d x} \leq \frac{8 k^{p+3}}{L^{2} \bar{\rho}},
$$

and the proof is finished.

\section{ACKNOWLEDGEMENTS}

This work was partially supported by Universidad de Buenos Aires under grant 20020130100283BA, ANPCyT PICT2012 0153, and by CONICET (Argentina) PIP $5478 / 1438$.

\section{REFERENCES}

[1] W. Allegretto, Y. X. Huang, Eigenvalues of the indefinite weight p-Laplacian in weighted spaces. Funkcial Ekvac. 38 (1995) 233-242.

[2] G. Borg, On a Liapunoff criterion of stability, Amer. J. Math. 71 (1949), 67-70.

[3] T. Champion, L. De Pascale, Asymptotic behaviour of nonlinear eigenvalue problems involving p-Laplacian-type operators, Proc. Roy. Soc. Edinburgh Sect. A 137 (2007), no. 6, 1179-1195.

[4] D. Cioranescu. P. Donato, An introduction to homogenization, Oxford Lecture Series in Mathematics and its Applications, vol. 17, The Clarendon Press Oxford University Press, New York, 1999. MR 1765047 (2001j:35019).

[5] M. Cuesta, Eigenvalue problems for the p-Laplacian with indefinite weights. Electronic Journal of Differential Equations, 2001 (33) (2001) 1-9.

[6] K. M. Das, A. S. Vatsala, Green's function for $n-n$ boundary value problem and an analogue of Hartman's result, J. Math. Anal. Appl. 51 (3) (1975) 670-677. 
[7] J. Fernández Bonder, J. P. Pinasco, Eigenvalues of the p-Laplacian in fractal strings with indefinite weights, Journal of Mathematical Analysis and Applications 308 (2005) 764-774.

[8] J. Fernández Bonder, J.P. Pinasco, A.M. Salort, Convergence rate for some quasilinear eigenvalues homogenization problems. Journal of Mathematical Analysis and Applications 423 (2015) 1427-1447.

[9] J. Fernández Bonder, J. P. Pinasco, A. M. Salort, Some results on quasilinear eigenvalue problems, to appear in Rev. Matemática de la UMA.

[10] J. Fleckinger, M. L. Lapidus, Eigenvalues of elliptic boundary value problems with an indefinite weight function. Transactions of the American Mathematical Society 295 (1986) 305-324.

[11] J. García Azorero, I. Peral Alonso, Comportement asymptotique des valeurs propres du p-laplacien, C. R. Acad. Sci. Paris Sér. I Math. 307 (1988), no. 2, 75-78. MR 954263 (89k:35171).

[12] B. J. Harris, Q. Kong, On the oscillation of differential equations with an oscillatory coeffcient, Trans. Amer. Math. Soc. 347 (1995) 1831-1839.

[13] H-L. Hong, W-C. Lian, C. C. Yeh, The oscillation of half-linear differential equations with an oscillatory coefficient, Mathematical and computer modelling 24 (1996) 77-86.

[14] P. Hess, T. Kato, On some linear and nonlinear eigenvalue problems with an indefinite weight function, Communications in Partial Differential Equations 5 (1980) 999-1030.

[15] T. Kilpelainen, Weighted Sobolev spaces and capacity, Ann. Acad. Sci. Fenn. Ser. A I Math. 19 (1994) 95-113.

[16] S. A. Nazarov, I. L. Pankratova, A. L. Piatnitski, Homogenization of the spectral problem for periodic elliptic operators with sign-changing density function, Archive for rational mechanics and analysis 200 (2011) 747-788.

[17] B. Opic, A. Kufner, Hardy-type inequalities. Longman Group UK, 1990.

[18] I. Pankratova, A. Piatnitski, Homogenization of spectral problem for locally periodic elliptic operators with sign-changing density function, Journal of Differential Equations 250 (2011) 3088-3134.

[19] J. P. Pinasco, Lower bounds for eigenvalues of the one-dimensional p-Laplacian, Abstract and Applied Analysis 2004 (2004) 147-153.

[20] J. P. Pinasco, Lyapunov type inequalities, with applications to eigenvalue problems, Springer Briefs in Mathematics, Springer, New York, 2013.

[21] D. Smets, A Concentration-Compactness Lemma with Applications to Singular Eigenvalue Problems Journal of Functional Analysis 167 (1999) 463-480.

[22] B. O. Turesson, Nonlinear potential theory and weighted Sobolev spaces. Lecture Notes in Math. 1736. Springer, 2000.

[23] K. Watanabe, Lyapunov type inequality for the equation including 1-dim p-Laplacian, Mathematical Inequalities \& Applications 15 (3) (2012) 657-662.

[24] A. Wintner, On the Non-Existence of Conjugate Points. American Journal of Mathematics 73 (1951) 368-380.

[25] E. Zeidler, Lectures on Lyusternik-Schnirelman theory for indefinite nonlinear eigenvalue problems and its applications. In: Nonlinear analysis, function spaces and applications (Proc. Spring School, Horni Bradlo, 1978), pp. 176219, Teubner, Leipzig, 1979. Available at http: //dml.cz/dmlcz/702410. MR 0578914.

Departamento de Matemática

IMAS - CONICET

FCEyN - Universidad de Buenos Aires

Ciudad Universitaria, Pabellón I

(1428) Av. Cantilo s/n.

Buenos Aires, Argentina.

E-mail address, J. Fernandez Bonder: jfbonder@dm.uba.ar

URL, J. Fernandez Bonder: http://mate.dm.uba.ar/ jfbonder

E-mail address, J.P. Pinasco: jpinasco@dm.uba.ar

URL, J.P. Pinasco: http://mate.dm.uba.ar/〜jpinasco

E-mail address, A.M. Salort: asalort@dm.uba.ar 